Pp. $1-7$

DOI: 10.1089/end.2019.0686

\title{
Nanostructured Gold Coating for Prevention of Biofilm Development in Medical Devices
}

\author{
MS \\ PhD \\ PhD \\ PhD \\ PhD

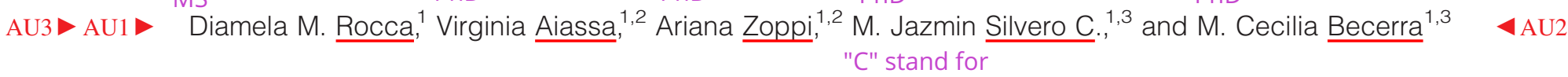 \\ Compagnucci \\ However, my ORCID \\ Abstract \\ name appears as $\mathrm{M}$. \\ Jazmin Silvero C.
}

Bacterial biofilms on medical devices (MDs) can cause deadly infections due to their resistance to antibiotics. Technology to prevent this kind of complication is urgently needed because they impact not only patients' lives but also hospital budgets. In this article, the creation and testing of an easy-to-produce antibiofilm (more precisely antibiofouling) coating are described for the first time. This coating can be applied to catheters, prostheses, and other plastic pieces, even after they have been manufactured. Rapid and ecofriendly synthesis of nanostructured gold coating was done in situ in just 15 minutes. Complete characterization and microbiological analysis of its antibiofouling capacity are presented. The coating prevents biofilm formation of pathogenic clinical isolates and ATCC strains on MDs, possibly due to its complex nanostructured gold surface. If the next generation of MDs is coated with this kind of antibiofouling technology, biofilm-related infections could be dramatically reduced.

AU6 Graphical Abstract

Graphical abstract

OK

\section{Nanostructured Gold Coating for Prevention of Biofilm Development in Medical Devices}

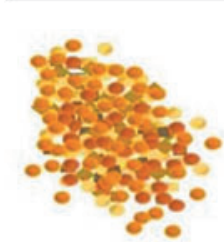

AuHCl$\cdot 3 \mathrm{H}_{2} \mathrm{O}$
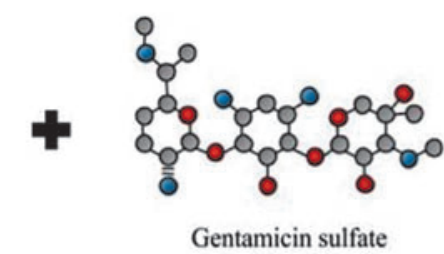

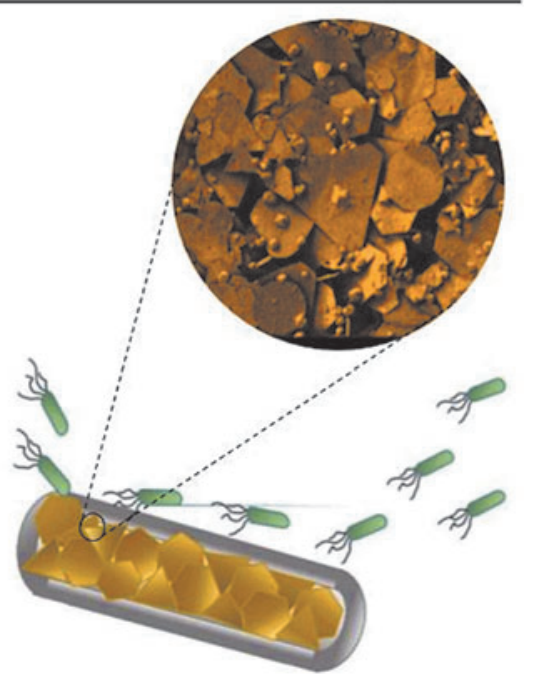

Antifouling capacity of the coating

$4 \mathrm{C}$

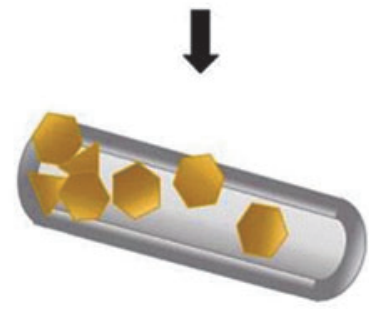

Coating of catheter

Au7. Rapid and ecofriendl

Conclusion: Synthesis of nanostructured gold Reference: Diamela M. Rocca, Virginia Aiassa, Ariana Zoppi, coating was done in situ inside a catheter in $15 \mathrm{~min}$. This coating prevents biofilm formation of pathogenic clinical isolates.
Reference: Diamela M. Rocca, Virginia Aiassa,
M. Jazmin Silvero C., and M. Cecilia Becerra Dol: 10.1089/end 2019.0686 AU8: "C" stands for Compagnuccinolusever my ORCID name is M. Jazmin Silvero C.

\footnotetext{
${ }^{1}$ Departamento de Ciencias Farmacéuticas, Facultad de Ciencias Químicas, Universidad Nacional de Córdoba, Córdoba, Argentina. AU4 ${ }^{2}$ Unidad de Investigación y Desarrollo en Tecnología Farmacéutica - UNITEFA, CONICET. Córdoba, Argentina ${ }^{3}$ Instituto Multidisciplinario de Biología Vegetal - IMBIV, CONICET. Córdoba, Argentina
} 
AU5 Keywords: coating, nanomaterial, biofilm, antibiofouling, antibacterial, gold

\section{Introduction}

C URRENTLY, BIOFILMS ARE of major importance in hospital infections, especially those related to the implantation of medical devices (MDs). Biofilms are aggregates of microorganisms embedded within a self-produced extracellular polymeric matrix that makes them resistant to external aggression. The majority of currently available antibiotics do not penetrate this biofilm and the infection continues. The best strategy is to prevent the adhesion of the first cells to the surface of MDs before the biofilm is able to develop. ${ }^{1}$ Recent reports estimated that 4,100,000 patients are affected by this kind of infection in Europe and 1,737,125 in the United States. The number of associated deaths is up to 95,000 . Interestingly, an analysis of the costs in the U.S. indicates that if hospitals were to prevent biofilm formation on the MDs they use, they could save thousands of dollars annually. ${ }^{2}$ Therefore, the development of effective antibiofouling options is urgent.

Ideal antibiofouling material could also be applied in other areas, from the food industry to everyday surfaces. It has been known for many years that gold is an incorruptible material and has been used for medical instruments and prosthesis. Indeed, as it is biologically benign, it features numerous advantages when used in medical catheters, stents, implants, instruments, and devices. ${ }^{3,4}$ However, with the rise of polymer science, this has been replaced by other much cheaper and flexible materials (such as silicone). These materials are very susceptible to bacterial attachment, therefore, there have been many attempts to coat them with noble metals like gold The cost of pure solid gold and the time-consuming process of racking or wiring (electroplating), and the toxic reactants like chromic acid, prevent the massive use of gold-plated MDs. As such, this is the first time that a procedure to coat any plastic MD in less than 30 minutes, using a minimum quantity of reactants, has been described. Moreover, this novel coating is actually made of nanostructured gold, which has a higher capacity to prevent microorganism attachment. This nanostructured gold coating was developed through the reduction of gold salt using the carefully selected aminoglycoside gentamicin. It is worth noting that gentamicin does not remain bonded to gold nano plate (GNP); it only acts as a precursor for their formation.

\section{Materials and Methods}

Materials

The gold salt $\mathrm{AuHCl}_{4} \cdot 3 \mathrm{H}_{2} \mathrm{O}$, glucose, and $\mathrm{D}_{2} \mathrm{O} 99.9$ atom\% D, were purchased from Sigma-Aldrich. Water, intended for use in all analyses, was obtained from a Millipore Milli-Q water purification system. Gentamicin sulfate was purchased from Todo Droga (Argentina). Solvents were of analytical reagent grade and were acquired from Cicarelli (Argentina).

\section{Equipment}

All ultraviolet (UV)-visible spectra were collected employing a Biotek (CIBICI-CONICET -Dpto. Bioquímica
Clínica-FCQ-UNC). The ${ }^{1} \mathrm{H}-\mathrm{RMN}$ spectra were recorded in $\mathrm{D}_{2} \mathrm{O}$ using a High-Resolution Bruker Avance II-400 spectrometer at (INFIQC-CONICET - Dpto. Qca. Orgánica FCQ-UNC). The scanning electron microscopy/energy dispersive X-ray spectrometry (SEM/EDS) — Carl Zeiss-Sigma and the DRX-Philips-PW 1800 were from LAMARX-IFEG Laboratory (FAMAF-UNC).

\section{Coating and characterization}

The $\mathrm{Au}^{3+}$ solution $(0.1 \mathrm{M})$ and the gentamicin solution $(1.0 \mathrm{M})$ were prepared by directly dissolving the solids $\left(\mathrm{AuHCl}_{4} \cdot 3 \mathrm{H}_{2} \mathrm{O}\right.$ and gentamicin sulfate) in MQ water. Next, equal quantities of each solution were placed dropwise in a Falcon tube containing MQ water. The mixture was shaken in a vortex between drops. The final solution was heated for 15 minutes in a thermostatic water bath. Microwave heating with a regular appliance was also tested (heated for three cycles of 10 seconds each, leaving the mixture to rest at room temperature for 30 seconds in between). All solutions were freshly prepared before the synthesis and allowed to stabilize at room temperature for $1 \mathrm{~min}$ before heating. The GNPs obtained were characterized using SEM, EDS, and X-ray diffraction (XRD). Statistical analysis of size, and shape analysis of GNP were performed using Image ${ }^{\circledR}$ software. ${ }^{5}$ RMN was applied to evaluate gentamicin in the supernatant. 4 AU9 "RMN" should be replaced with english abbreviation "NMR"

Coating of catheters/prosthesis AU10: "NMR" stands for Nuclear Magnetic Resonance

Homogeneous deposition of GNP on a silicone urinary catheter was performed by applying the procedure detailed above with minor changes. Briefly, $100 \mu \mathrm{L}$ of $\mathrm{HAuCl}_{4}$ $(0.1 \mathrm{M})$ was added to $900 \mu \mathrm{L}$ of MQ water and $100 \mu \mathrm{L}$ of gentamicin $(1.0 \mathrm{M})$. The catheter was filled with the solution and heated in a thermostatic bath at $70^{\circ} \mathrm{C}$ for 15 minutes. Finally, catheters were washed twice with MQ water. For prostheses, complete immersion is required.

\section{Durability and resistance of the coating}

This kind of catheter is usually submitted to liquid erosion, dropwise or by constant flux. The durability and resistance of the coating were evaluated in the laboratory simulating the conditions of real use for further clinical purposes. The physiological solution $(500 \mathrm{~mL})$ was syringe pumped inside coated catheter samples (triplicates), according to Hurst et al. with some modifications. ${ }^{6}$ Pictures were taken employing an optical microscope $(10 \times, 40 \times$, and $100 \times)$, before and after liquid erosion, and corroborate the SEM images. However, optical microscopy has the advantage of being a lessexpensive method for testing the coating when this process is scaled up for industrial manufacturing. ImageJ software was used to quantify the coated surface area.

\section{Antibiofouling capacity of the coating}

Samples of coated and uncoated silicone catheters ("Admisol dropper" from Gavamax ${ }^{\circledR}$ laboratories, and "Rusch Gold Two-Way Silicone-Coated Latex Foley Catheter" from Teleflex Medical), containing natural rubber latex, were "NMR" stands for Nuclear Magnetic Resonance 


\section{NANOSTRUCTURED ANTIBIOFOULING COATING}

incubated with three biofilm-forming bacterial strains: two clinical strains: methicillin-resistant Staphylococcus aureus 9455 (MRSA 9455) and Pseudomonas aeruginosa 191150, and a control strain, Escherichia coli ATCC 25922. Catheter samples were prepared by cutting them in half lengthwise (length $1 \mathrm{~cm}$, diameter $0.015 \mathrm{~cm}$ ) using a sterile sharp scalpel. All the samples were UV sterilized for 30 minutes before the incubation (15 minutes on each side). Following this, samples were incubated in $2 \mathrm{~mL}$ of Tryptic soy broth supplemented with $0.25 \%$ glucose (to promote biofilm formation) inoculated with the bacterial suspensions $\left(10^{6} \mathrm{CFU} / \mathrm{mL}\right)$ for 18 hours under shaking at $130 \mathrm{rpm}$, and samples were removed and washed twice with phosphate-buffered solution to remove planktonic bacteria. ${ }^{7}$ The samples were then dehydrated with increasing ethanol percentages $(50 \%, 70 \%, 80 \%$, $90 \%$, and $100 \%$ ) for 15 minutes in each solution, and then dried at room temperature. For SEM/EDS analysis, each sample was gold sputter coated and mounted on a carbon film. The magnification selected was sufficient to appreciate in detail the general morphology of the samples under study. EDS was used to determine the distribution of GNP and to differentiate the gold particles from bacteria. Formation of biofilm upon the surface of raw catheters and coated catheters was measured using ImageJ software.

\section{Results}

\section{Coating and characterization}

The purpose of this experiment was to coat different plastic catheters/prosthesis with a gold laminar nanoplate antibiofouling coating that would prevent the formation of bacterial biofilms using a quick and ecofriendly method.

As a starting point, the synthesis was tested in Falcon tubes. After mixing the two reactants, the initial pale yellow color due to the gold salt disappeared, and the solution became colorless after 5 minutes of heating. Following this, bright gold crystals started to appear as the solution turned goldish and the Falcon walls acquired a coppery color.
Finally, a large amount of a bright gold solid was observed while shaking the solution (Supplementary Fig. S1).

Identical results were observed when heating the samples for 60 seconds in a conventional microwave. After this, to obtain a solid product as pure as possible, the Falcon content was transferred to a clean Eppendorf and centrifuged at $3500 \mathrm{rpm}$. After 45 minutes, a bright gold precipitation (6.82 $\mathrm{mg}, 87 \%$ yield) was obtained at the bottom of a colorless liquid (Supplementary SI. Fig. S2).

The bright gold solid (pellet) was completely submerged in concentrated nitric acid and suffered no visible changes, suggesting the presence of the expected noble metal. The purity of this solid was evaluated through EDS (Supplementary Fig. S3). The composition was found to be $100 \%$ $\mathrm{Au}^{0}$. To conclude, XRD reveals a face-centered cubic crystalline structure with the characteristic peaks at $2 \Theta: 38,44$, 64,78 , and 81 assigned to the lattice planes 11, 200, 220,311, and 222, respectively (Supplementary Fig. S4). This was as 4 SF4 expected for metallic gold. The supernatant had a $\mathrm{pH}$ of 1 , whereas the initial solution had a pH of 5 .

\section{Coating of plastic catheters/prosthesis}

Employing a slightly modified synthesis of the gold nanoplates mentioned above, the lumen of catheters (Fig. 1) and $4 \mathrm{~F} 1$ the surface of a silicone prosthesis (a section of a penile prosthesis) were completely coated in just 15 minutes (Fig. 2).

The EDS spectrum (Supplementary Fig. S5) shows the 4 SF5 predominant presence of gold $(72.6 \%)$, followed by silicon and oxygen, which are part of the silicone catheter wall.

\section{Durability and resistance}

Pictures taken before and after the liquid erosion test proved that the same amount of GNP remained attached to the catheter. ImageJ-processed pictures could be found in Figure 3.

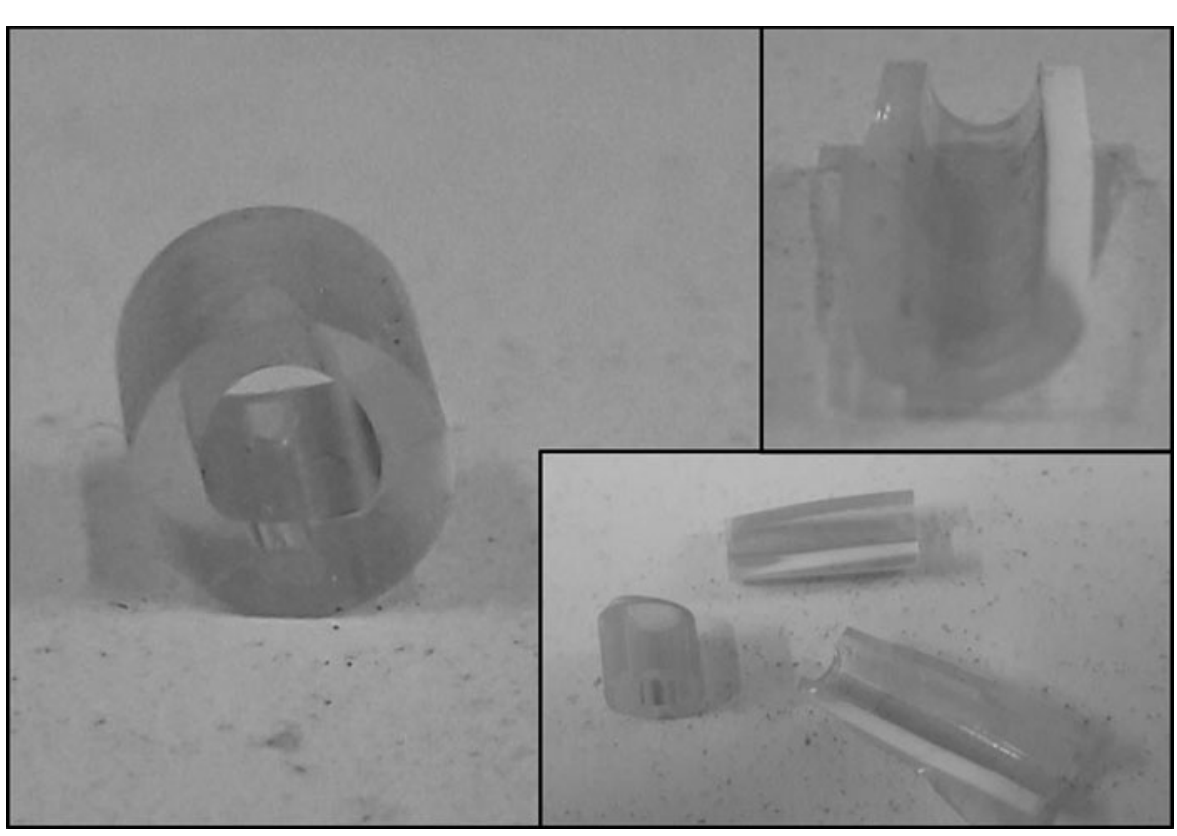

FIG. 1. Pictures of plastic catheters after coating and sliced samples for SEM imaging. SEM, scanning electron microscopy. 


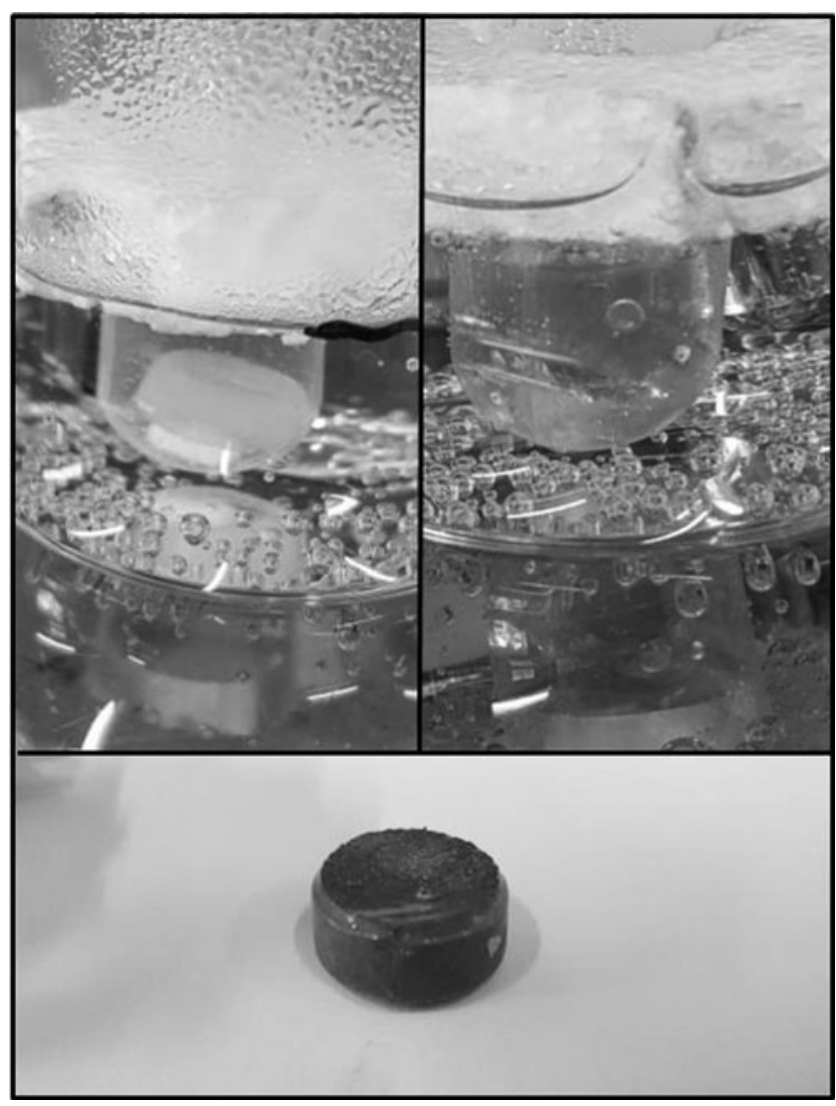

FIG. 2. Initial phase (top left) and final phase (top right) of the in situ coating on the surface of a prosthesis. The prosthesis is completely coated (bottom).

\section{Antibiofouling capacity of the coating}

In this study, it was observed that Gram-negative bacteria,

F4 such as P. aeruginosa (Fig. 4: 1) and E. coli (Fig. 4: 3), adhered to the catheter, whereas $S$. aureus (Fig. 4: 5) formed a biofilm. However, when the catheters had been previously coated none of the three strains under study was able to adhere to the plastic surface. In fact, in the microphotographs of

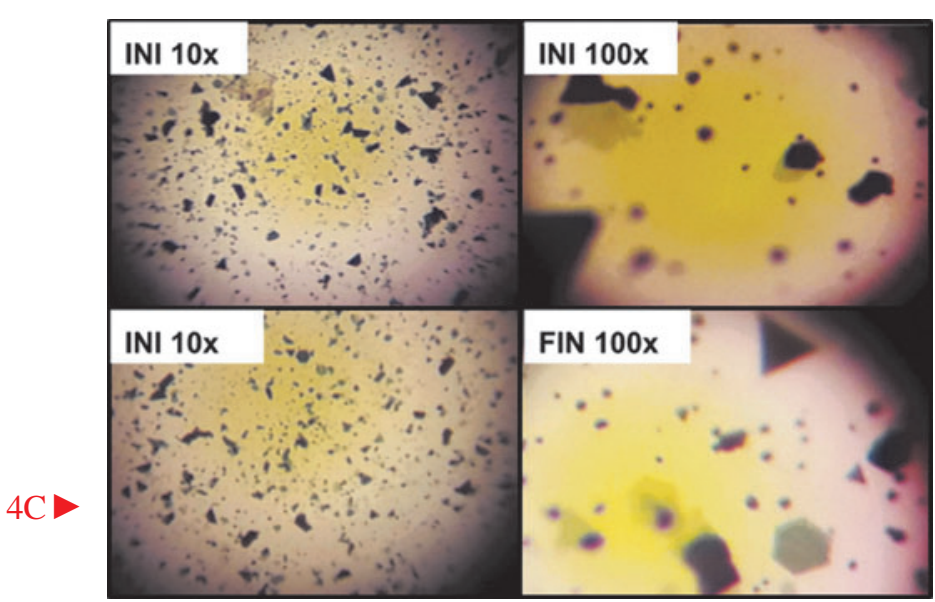

FIG. 3. Selected optical microscope images of coated silicone catheter before and after liquid erosion test. Labeled as initial (INI) and final (FIN). the coated catheters, only the gold microplates were observed even though they were incubated for 18 hours with bacteria inoculum (Fig. 4: 2, 4 and 6). Usually, biofilm formation begins after just 4 hours of exposition to a lower inoculum of microorganisms. Consequently, 18 hours would have been more than sufficient time for a mature biofilm to develop, considering the bacterial suspension of $1,000,000 \mathrm{CFU} / \mathrm{mL}$.

These results support the hypothesis that coated catheters prevent biofilm development of the three tested strains.

ImageJ processing of the SEM images confirms the effect against the bacterial adherence on the plastic surface. The Replace "SF7" percentage of bacteria covering the surface decreased while with "SF6" the percentage of gold nanoplates covering the surface increased (Supplementary Figure S7 and Supplementary 4 SF7 4 ST1 Table S1).

\section{Discussion}

There are several benefits to gold plating an MD, such as the ones mentioned on websites providing gold plating services in medicine: improving electrical conductivity of some MDs that require an electrical current to function, increasing thermal conductivity and therefore minimizing burns and the formation of adhesions, extending wear and corrosion resistance, facilitating biocompatibility, cleanliness and sanitation, and establishing a radiopaque area. ${ }^{8}$

It is known that nanometric gold offers unique qualities in contrast to the bulk form. Indeed, the coating of MDs with gold nanoplates (GNPs) has even more advantages than regular laminar coating. This could be explained by the many unique characteristics of nano-sized gold. ${ }^{9}$ This article explores the capacity to prevent biofilm formation, because this is one of the most common and serious problems relating to plastic MDs. ${ }^{10}$ Biofilms are highly organized microbial communities with functional heterogeneity that are commonly formed on natural and synthetic surfaces. They protect microorganisms from the harsh external environment through self-produced matrices of extracellular polymeric substances. ${ }^{11}$ Moreover, they are also more resistant to antimicrobial agents than the same microorganism grown in a free-swimming or planktonic state and they are the main reason for the failure or removal of MDs. ${ }^{12}$ Other types of nanomaterials to prevent biofilm formation have been tested, but their synthesis involves several steps and reactants. ${ }^{13}$ The nanotopography of the surface is of major importance for the first steps of the bacterial adhesion, as described by Prosenjit Sen's research group. ${ }^{14-16}$ The major advantage of this coating is that there are only two reactants: $\mathrm{HAuCl}_{4}$ salt and gentamicin; a commercially available, broad-spectrum antibiotic.

Gentamicin was chosen because it is known to be a reducing aminoglycoside, due to the capability of its functional groups to be oxidized to carbonyls. This particular property was employed, in this case, to reduce $\mathrm{Au}^{+3}$ and form GNPs.

\section{Coating and characterization}

Gentamicin is an aminoglycoside antibiotic composed of purpurosamine, deoxystreptamine, and garosamine with variations in the substitution pattern of the purpurosamine Replace SF8" resulting in four major components: C1, C1a, C2, and C2a with "SF7" (Supplementary Fig. S8). Deubner and colleagues demon- $\varangle$ SF8 strated that NMR studies allow the characterization of the $\varangle$ AU10 "NMR" stands for Nuclear Magnetic Resonance 


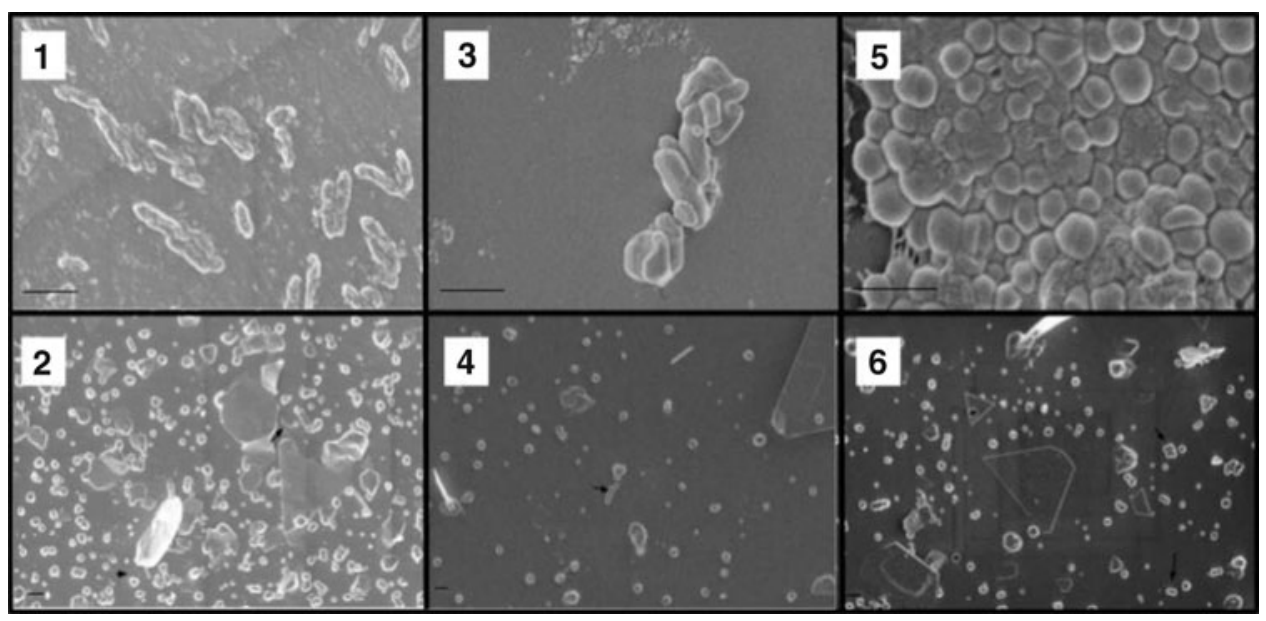

FIG. 4. SEM of catheters incubated with biofilmforming clinical bacterial strains: Pseudomonas aeruginosa $(\mathbf{1}, 2)$, Escherichia coli $(\mathbf{3}, \mathbf{4})$, and Staphylococcus aureus $(\mathbf{5}, \mathbf{6})$. Note how the bacteria were attached to the surface of uncovered catheters $(\mathbf{1}, \mathbf{3}, \mathbf{5})$, but not to the coated catheters $(\mathbf{2}, \mathbf{4}, \mathbf{6})$, where only a few planktonic forms of bacteria (arrows) could be observed.

composition of the main components, analyzing the area covered by the anomeric hydrogens of the purpurosamine part of the molecule. ${ }^{17}$

Therefore, the gentamicin used in this experiment was characterized using ${ }^{1}$ H NMR. The spectrum obtained showed the presence of the four major components in the sample (Supplementary Fig. S8). It is therefore suggested that gentamicin, due to its functional groups, is responsible for the reduction of the gold salt and the liberation of $\mathrm{HCl}$ to the supernatant. The strong acidity of the supernatant after the reaction was attributed to the $\mathrm{HCl}$ liberated during the reaction to the aqueous media of the gold and the antibiotic salts. Additionally, the presence of carbonyl groups was detected with 2, 4-dinitrophenylhydrazine.

Interestingly, the pure $\mathrm{Au}^{0}$ crystallized in thin laminar geometrical forms. Most are hexagonal, but triangular and other polyhedral shapes were also observed in SEM images

Replace "SF9" with "SF8"
(Supplementary Fig. S9), with sides from 2 to $30 \mu \mathrm{m}$. Ad ditionally, some amorphous clusters were found, which too seem to be formed by the smallest geometrical pieces. Distribution of shapes and sizes of the gold microplates can be SF6 AU11 seen in Supplementary Figure S6. Overall, the analysis of the Replace solid and the supernatant suggest that gentamicin is oxidized "SF6" with while reducing the $\mathrm{Au}^{3+}$ to $\mathrm{Au}^{0}$ through the following general "SF9" proposed reaction:

$$
\begin{aligned}
& \mathrm{Au}^{3+}{ }_{(\mathrm{aq})}+\text { gentamicin }_{(\mathrm{aq})} \rightarrow \\
& \mathrm{Au}_{(\mathrm{s})}^{0}+\text { oxidized gentamicin products }_{(\mathrm{aq})}
\end{aligned}
$$

\section{Coating of plastic catheters/prosthesis}

After this in situ synthesis inside the catheter, a coat of gold nanoplates cover the catheter lumen homogeneously and prevent bacteria from attaching (Fig. 8).

This method of synthesis offers a method to prepare a coat of pure GNP formed directly on the plastic surface (Figs. 1 and 2). The byproducts of the in situ reaction are nontoxic oxidized gentamicin and the gold salt counter ions that can be discarded like regular chemicals. Other early reports on the synthesis of GNP could be found, but they are limited by both low purity and yield. ${ }^{18,19}$

The prosthesis coating would require in vivo follow-up tests to ensure biocompatibility for long-term devices. These experiments are being planned.

\section{Durability and resistance}

The durability of the coating could be attributed to the favorable interaction between gold nanomaterials and silicone, which has been well established previously. In fact, there is a report on the noticeable role that proteins on the surface of the rubber latex play in the nucleation and stabilization of gold nanomaterials. ${ }^{20}$ This kind of positive interaction could explain why there were no significant changes in the coating when observed using an Optical Microscope (Fig. 3). Using this technique, the bigger GNPs could be easily observed. Their distribution was homogeneous and did not change or decrease with time. The advantage of OM is that it could be performed on a large number of samples immediately after they were tested for durability, the limitation being it lacks the capability to check the smallest nanoparticles.

Images of erosion tests indicate that there is no significant difference between the initial coated surface area and the final one $(80 \% \pm 2 \%$ and $78 \% \pm 1 \%$, respectively) This could suggest that this coating is resistant enough to be employed in regular clinical use.

\section{Antibiofouling capacity of the coating}

Biofilm development on plastic MDs and the subsequent infections they cause are among the primary reasons to gold coat them. A clinical study compared the bacteriuria (as a urethral infection indicator) of 217 patients treated with a silicone catheter, with 222 patients treated with a gold-coated latex catheter. It was found that noble metal alloy coating resulted in a five times reduction in catheter-associated infections. ${ }^{21}$ Another more recent article reports that noble metal alloy coating proved to be a good strategy in reducing adverse effects related to catheters, while maintaining a safe toxicological evaluation. ${ }^{22}$ However, no significant difference was found in the microbial colonization.

Liedberg and coworkers compared the colonization of different urinary catheter coatings by a clinical strain of $P$. aeruginosa after 10 minutes and 10 hours of incubation and found that silver alloy-coated discs prevented the biofilm growth on latex, Teflon, ${ }^{\circledR}$ and silicone materials. ${ }^{23}$ Moreover, 9 trials with 13,319 demonstrate that silver coating confers antimicrobial properties to urinary catheters. ${ }^{24}$ The catheter fouling can be induced by the accumulation and adhesion of bacteria on the surface, thereby increasing the surface roughness. ${ }^{25}$ The results of this project demonstrate the high 
capacity of the coating to prevent the development of biofilms. The coating could be effectively applied to different silicone MDs, but was not tested on latex-based ones. However, the use of the latter is decreasing due to the disadvantages when compared with medical-grade silicone. The chemical resistance and mechanical/thermal properties of silicone have made it the preferred material for the latest MDs.

The antibiofouling effect observed (Fig. 4) could be related to the strong influence on the topography at the nanoscale in the bacterial attachment. Bacteria cells adapt their orientation in an attempt to maximize contact area with the surface, to stabilize their attachment, as reported by Hsu et al. ${ }^{26}$ A more detailed analysis by Rizzello and associates demonstrated the significant modifications in the morphological, genetic, and proteomic response of $E$. coli. to the nanostructured surface. Micrographs (by atomic force microscopy and SEM) captured the variations in bacteria shape and characteristics, specifically the loss of type- 1 adherent fimbriae. ${ }^{27}$ Both studies, in line with the behavior found on the surface of this coating, indicate that a purely physical change, such as the nanostructuration on the surface of the material, plays an important role in the prevention of biofilm development. This could explain the antibiofouling advantages of nanotechnology-based coating.

\section{Conclusion}

In summary, this novel method facilitates the production of high-purity gold microplates, which can be applied directly to the desired surface quickly and easily. The coating obtained is resistant to erosion and heat and confers its antibiofouling capacity on the material. This coating could be used in a wide range of MDs to prevent biofilm formation and extend their lifetime. The interesting properties of this material could result in further research, and its clinical application could be widespread.

Furthermore, the described nanoplates could have a potential application as tip-enhanced Raman scattering substrates.

\section{Acknowledgments}

The authors would like to thank Dr. G Bonetto (CONICET) and Sebastián García (LAMARX) for spectra acquisition, and Promedon S.A. for kindly providing the silicone prosthesis.

\section{Author Disclosure Statement}

All authors have approved the final version of this article and have no conflicts to declare. Universidad Nacional de Córdoba and CONICET declare Argentinian patent in process \#20180103397.

\section{Funding Information}

This work was supported by SECyT-UNC and FONCyT, Argentina (grant number 0821-2014). D.M.R. holds a AU12 SECyT doctoral scholarship. M.J.S.C., A.Z., V.A., and

\section{Supplementary Material}

Supplementary Figure S1

Supplementary Figure S2

Supplementary Figure S3

Supplementary Figure S4

Supplementary Figure S5

Supplementary Figure S6

Supplementary Figure S7

Supplementary Figure S8

Supplementary Figure S9

Supplementary Table S1

\section{References}

AU13

1. Almog M, Yanovskay A, Edelstein H, Schwartz N, Colodner R, Chazan B. Increasing antimicrobial resistance in long-term care facility patients with bacteremia: A 5 year's surveillance. J Am Med Dir Assoc 2018;19:1024-1026.e1.

2. Scott RD. The direct medical costs of healthcare-associated infections in US hospitals and the benefits of prevention. Division of Healthcare Quality Promotion National Center for Preparedness, Detection, and Control of Infectious Diseases Coordinating Center for Infectious Diseases Centers for Disease Control and Prevention March 2009. Available at www.cdc.gov/ncidod/dhqp/pdf/Scott_Cost Paper.pdf Last view Nov 1st 2019

3. Yachia D, Lask D, Rabinson S. Self-retaining intraurethral stent: An alternative to long-term indwelling catheters or surgery in the treatment of prostatism. Am J Roentgenol 1990;154:111-113.

4. Song J, Hu H, Jian C, Wu K, Chen X. New generation of gold nanoshell-coated esophageal stent: Preparation and biomedical applications. ACS Appl Mater Interfaces 2016; 8:27523-27529.

5. Rueden CT, Schindelin J, Hiner MC. ImageJ2: ImageJ for the next generation of scientific image data. BMC Bioinformatics 2017;18:529.

6. Hurst KM, Ansari N, Roberts CB, Ashurst WR. Selfassembled monolayer-immobilized gold nanoparticles as durable, anti-stiction coatings for MEMS. J Microelectromech Syst 2011;20:424-435.

7. Aiassa V, Zoppi A, Becerra MC, Albesa I, Longhi MR. Enhanced inhibition of bacterial biofilm formation and reduced leukocyte toxicity by chloramphenicol: $\beta$-cyclodextrin: N -acetylcysteine complex. Carbohydr Polym 2016; 152:672-678.

8. http://www.sharrettsplating.com/industries/medical-devices- 4 AU15 implants-plating

9. Major TA, Devadas MS, Lo SS, Hartland GV. Optical and Sharretts Plating dynamical properties of chemically synthesized gold na- Company, Inc. noplates. J Phys Chem C 2013;117:1447-1452.

10. Stickler DJ. Clinical complications of urinary catheters Last view Nov 1st caused by crystalline biofilms: Something needs to be done. 2019 J Intern Med 2014;276:120-129.

11. Garrett TR, Bhakoo M, Zhang Z. Bacterial adhesion and biofilms on surfaces. Prog Nat Sci 2008;18:1049-1056.

12. Costerton, JW. Bacterial biofilms: A common cause of persistent infections. Science 1999;284:1318-1322.

13. Mcconoughey SJ, Howlin R, Granger JF, Manring MM, Calhoun JH, Shirtliff M, Kathju S, Stoodley P. Biofilms in periprosthetic orthopedic infections. Future Microbiol 2014:9:987-1007.

14. Tripathy A, Kumar A, Chowdhury AR, Karmakar K, Purighalla S, Sambandamurthy V, Chakravortty D, Sen P. A nanowire-based flexible antibacterial surface reduces the viability of drug-resistant nosocomial pathogens. ACS Appl Nano Mater 2018;1:2678-2688. 
15. Tripathy A, Pahal S, Mudakavi RJ, Raichur AM, Varma MM, Sen P. Impact of bioinspired nanotopography on the antibacterial and antibiofilm efficacy of chitosan. Biomacromolecules 2018;19:1340-1346.

16. Tripathy A, Sreedharan S, Bhaskarla C, Majumdar S, Peneti SK, Nandi D, Sen P. Enhancing the bactericidal efficacy of nanostructured multifunctional surface using an ultrathin metal coating. Langmuir 2017;33:12569-12579.

17. Deubner R, Schollmayer C, Wienen F, Holzgrabe U. Assignment of the major and minor components of gentamicin for evaluation of batches. Magn Reson Chem 2003;41:589598.

18. Malikova N, Pastoriza-Santos I, Schierhorn M, Kotov NA, Liz-Marzán LM. Layer-by-layer assembled mixed spherical and planar gold nanoparticles: control of interparticle interactions. Langmuir 2002;18:3694-3697.

19. Sarma TK, Chattopadhyay A. Starch-mediated shapeselective synthesis of au nanoparticles with tunable longitudinal plasmon resonance. Langmuir 2004;20:3520-3524.

20. Gong W, He D, Tao J, Zhao P, Kong L, Luo Y, Peng Z, Wang H. Formation of defects in the graphite oxidization process: A positron study. RSC Adv 2015;5:88908-88914.

21. Stenzelius K, Persson S, Olsson U-B, Stjärneblad M. Noble metal alloy-coated latex versus silicone Foley catheter in short-term catheterization: A randomized controlled study. Scand J Urol Nephrol 2011;45:258-264.

22. Björling G, Johansson D, Bergström L, Strekalovsky A, Sanchez J, Frostell C, Kalman S. Evaluation of central venous catheters coated with a noble metal alloy-A randomized clinical pilot study of coating durability, performance and tolerability. J Biomed Mater Res B Appl Biomater 2017;106:2337-2344.

23. Liedberg H, Ekman P, Lundeberg T. Pseudomonas aeruginosa: Adherence to and growth on different urinary catheter coatings. Int Urol Nephrol 1990;22:487-492.

24. Johnson JR, Kuskowski MA, Wilt TJ. Systematic Review: Antimicrobial Urinary Catheters To Prevent CatheterAssociated Urinary Tract Infection in Hospitalized Patients. Ann Intern Med 2006;144:116.

25. Mukherjee $\mathrm{M}$, De S. Investigation of antibiofouling and disinfection potential of chitosan coated iron oxide-PAN hollow fiber membrane using Gram-positive and Gramnegative bacteria. Mater Sci Eng C 2017;75:133-148.
26. Hsu LC, Fang J, Borca-Tasciuc DA, Worobo RW, Moraru CI. Effect of micro- and nanoscale topography on the adhesion of bacterial cells to solid surfaces. Appl Environ Microbiol 2013;79:2703-2712.

27. Rizzello L, Sorce B, Sabella S, Vecchio G, Galeone A, Brunetti V, Cingolani R, Pompa PP. Impact of nanoscale topography on genomics and proteomics of adherent bacteria. ACS Nano 2011;5:1865-1876.

$$
\begin{gathered}
\text { Address correspondence to: } 4 \text { AU17 } \\
\text { PhDM. Jazmin Silvero C. } 4 \text { AU18 } \\
\text { Departamento de Ciencias Farmacéuticas "C" stands fro } \\
\text { Facultad de Ciencias Químicas Compagnucci. } \\
\text { Universidad Nacional de Córdoba However my } \\
\text { Haya de la Torre y Medina Allende ORCID name is "M. } \\
\text { Ciudad Universitaria Jazmin Silvero C." } \\
\text { Córdoba X5000HUA } \\
\text { Argentina }
\end{gathered}
$$

E-mail: jazmincompagnucci@gmail.com

PhD M. Cecilia Becerra Departamento de Ciencias Farmacéuticas Facultad de Ciencias Químicas Universidad Nacional de Córdoba Haya de la Torre y Medina Allende Ciudad Universitaria Córdoba X5000HUA Argentina

E-mail: becerra@fcq.unc.edu.ar

\begin{aligned} \multicolumn{1}{c|}{ Abbreviations Used } \\ $\mathrm{EDS}=$ energy-dispersive X-ray spectrometry \\ $\mathrm{GNP}=$ gold nanoplate \\ $\mathrm{MDs}=$ medical devices \\ $\mathrm{NMR}=$ Nuclear Magnetic Resonance \\ $\mathrm{SEM}=$ scanning electron microscopy \\ $\mathrm{UV}=$ ultraviolet \\ $\mathrm{XRD}=\mathrm{X}$-ray diffraction \end{aligned}




\section{Supplementary Data}

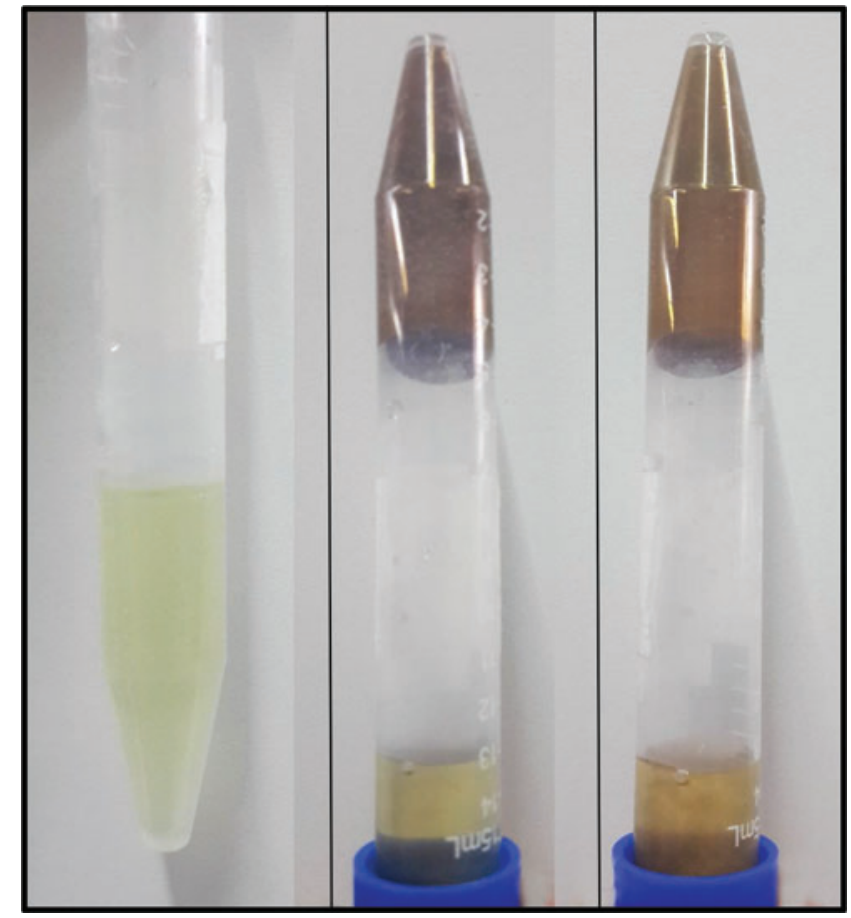

SUPPLEMENTARY FIG. S1. An initial pale yellow solution, before heating (left). Liquid with golden solid in suspension and coppery walls (middle). Final suspension with a large amount of golden solid (right).

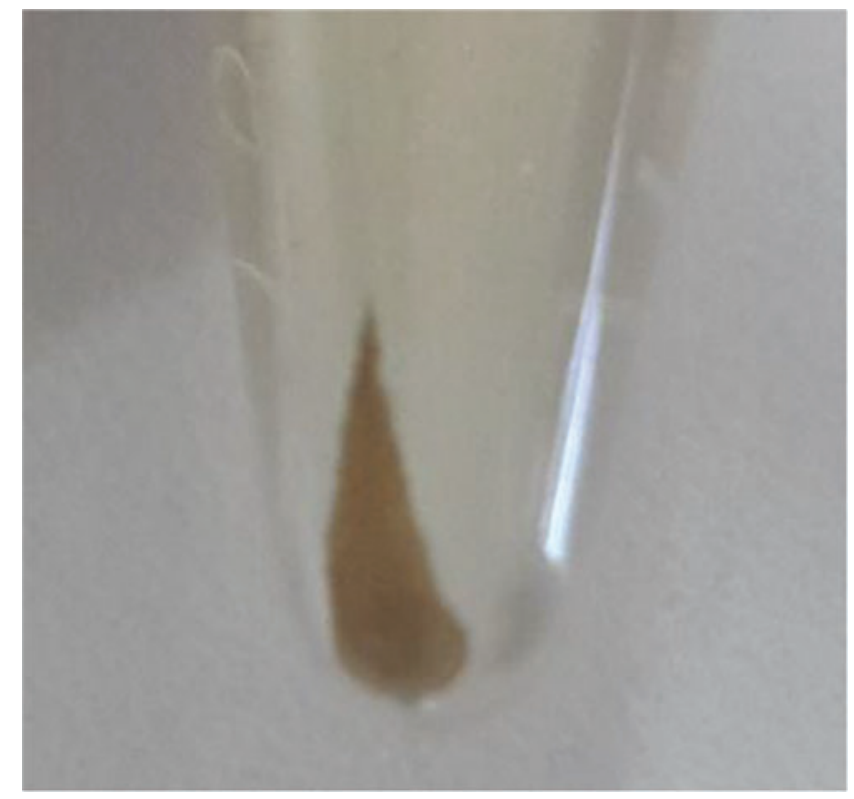

SUPPLEMENTARY FIG. S2. Bright gold solid precipitated after centrifugation at $3500 \mathrm{rpm}$.

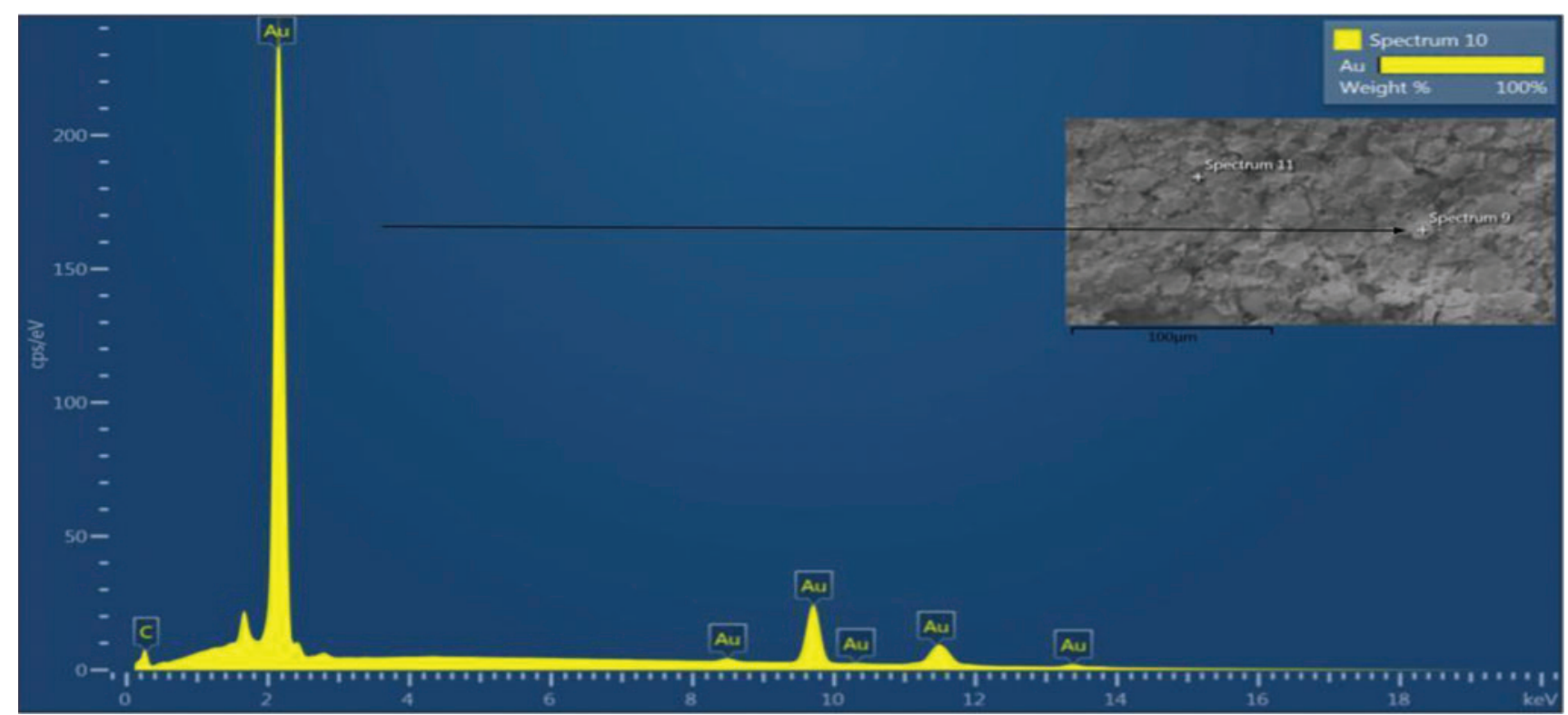

SUPPLEMENTARY FIG. S3. EDS spectrum of pure metallic gold obtained after reduction of the auric salt with gentamicin and its macroscopic view (insert). EDS, energy-dispersive X-ray spectrometry. 


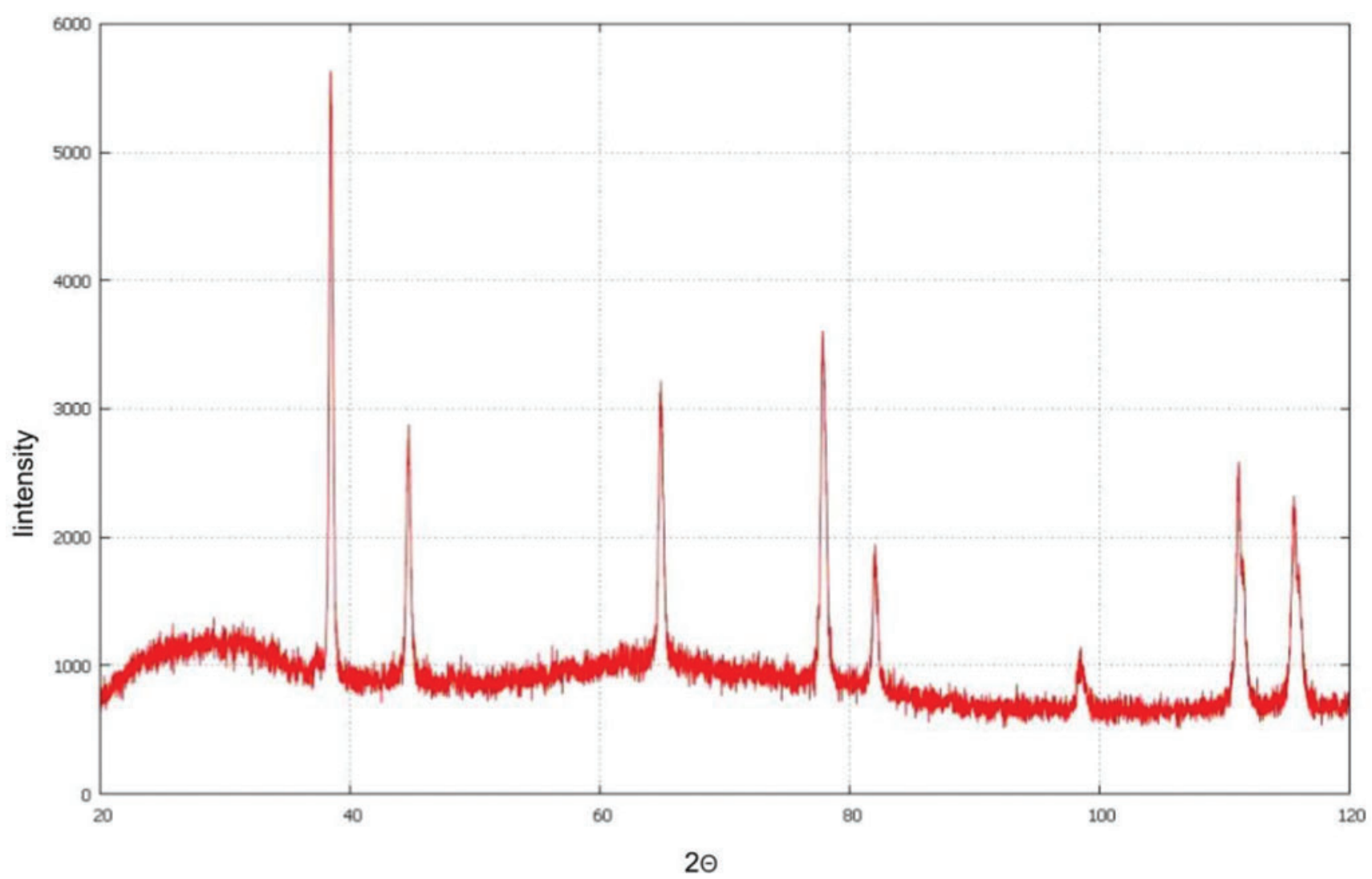

SUPPLEMENTARY FIG. S4. XRD pattern reveals a face-centered cubic crystalline structure characteristic of pure gold. XRD, X-ray diffraction.

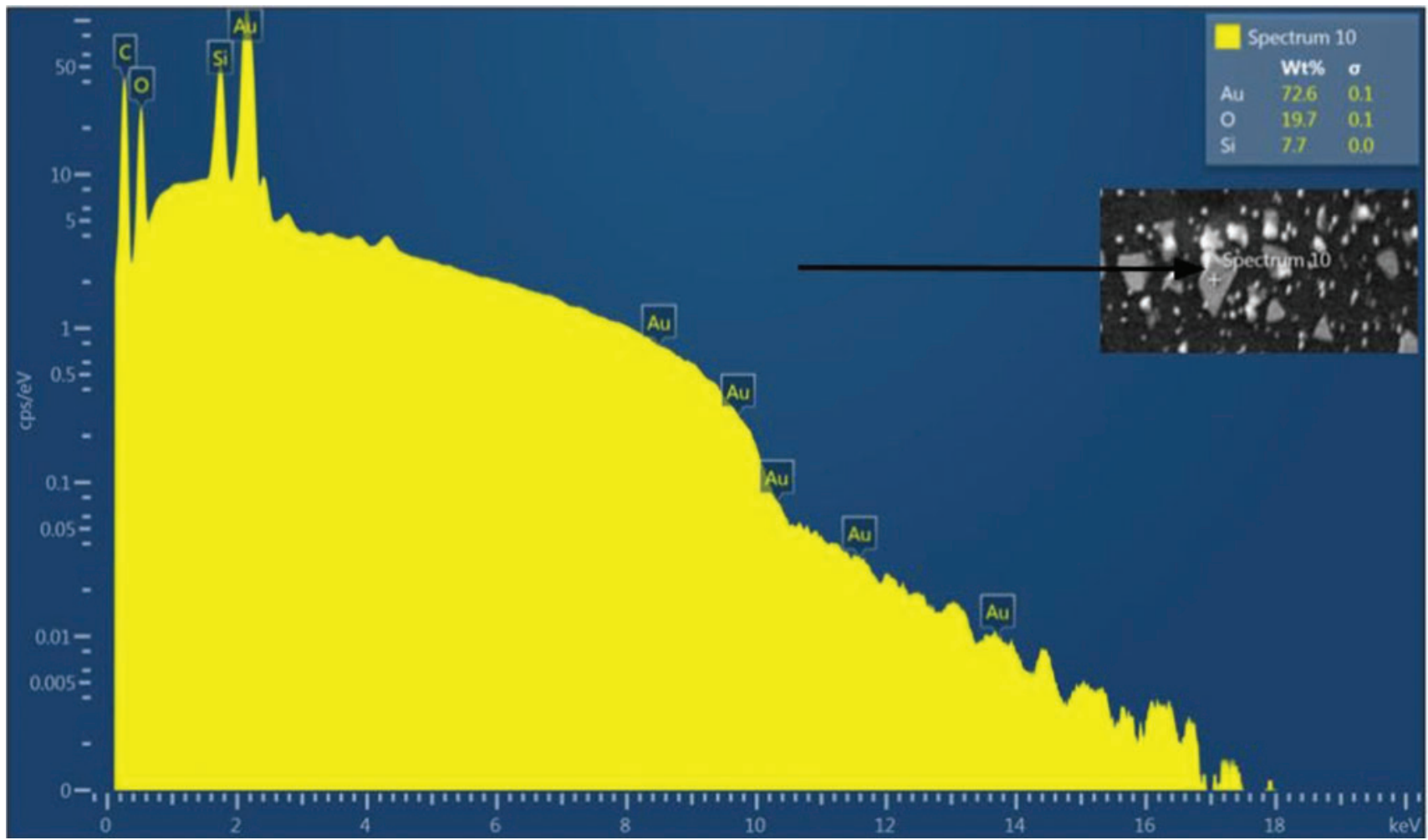

SUPPLEMENTARY FIG. S5. SEM/EDS of the catheter lumen after the coating. SEM, scanning electron microscopy. 


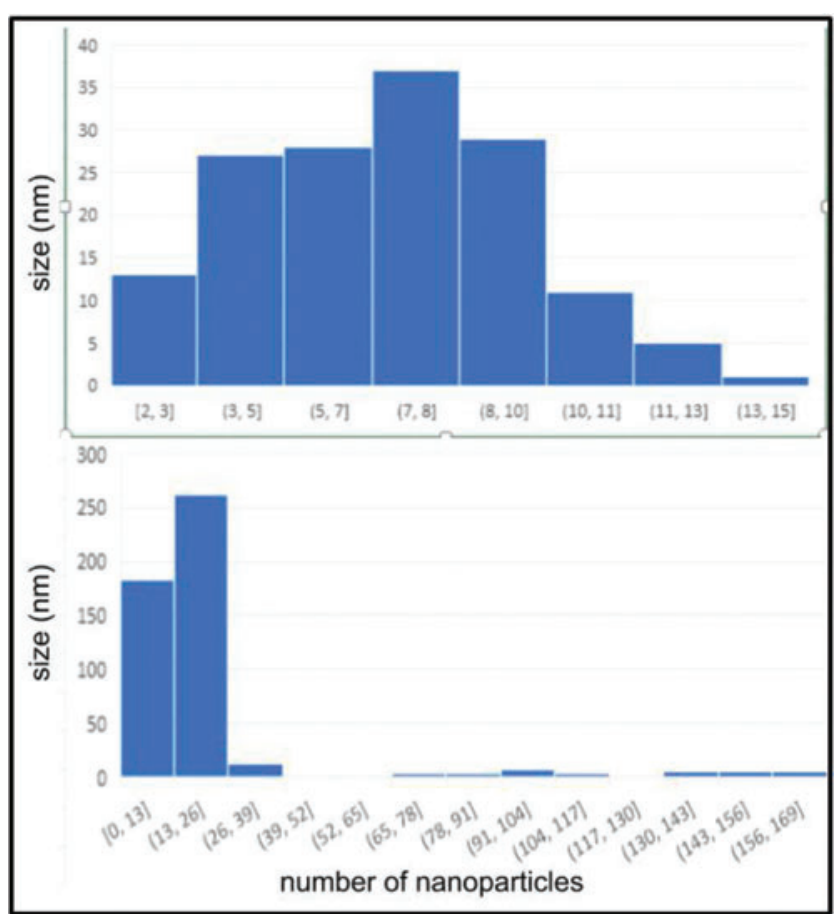

Replace "SF6" with SUPPLEMENTARY FIG. S6. Statistical distribution of "SF9" and rearrange its ubication side lengths (horizontal axis, nm) of hexagonal (top) and triangle (bottom) gold nanoplates.

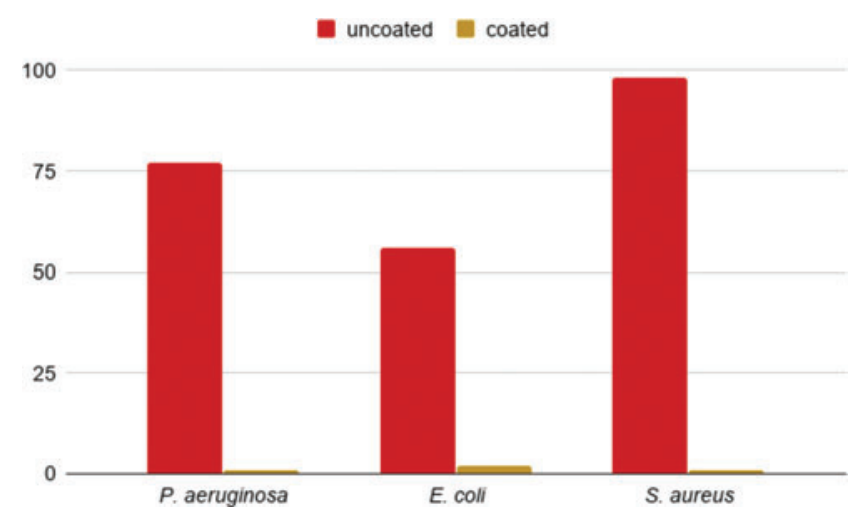

SUPPLEMENTARY FIG. S7. Antifouling effect of Replace "SF7" with the coating of a real catheter incubated with clinical "SF6" and antibiotic-resistant strains (A: Pseudomonas aeruginosa; B: rearrange its Escherichia coli; C: Staphylococcus aureus) expressed as ubication \%bacteria/\%coated surface of plastic catheter samples. SD $<4 \%$.
Replace "SF8" with "SF7" and rearrange its uhication

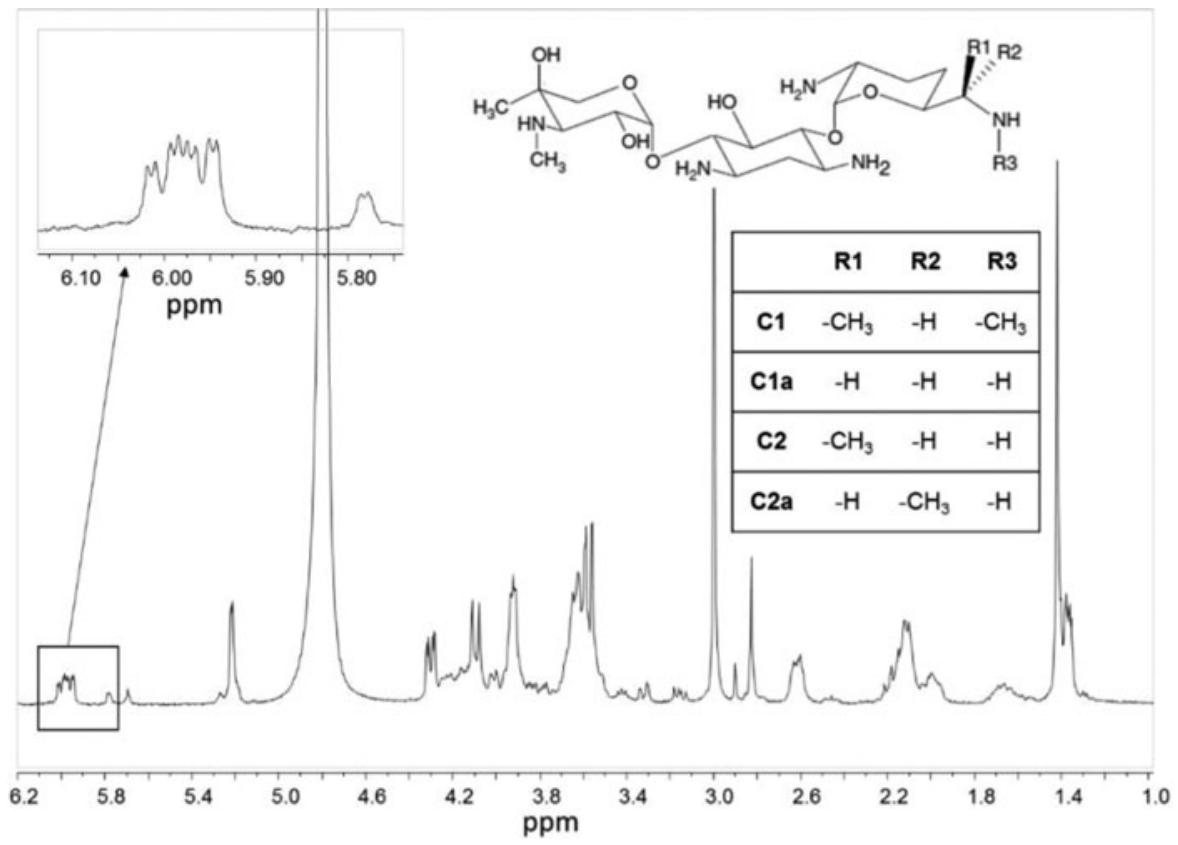

SUPPLEMENTARY FIG. S8. 1H NMR spectrum of gentamicin with an expansion of the region of anomeric hydrogen resonance signals of the different gentamicin components. 


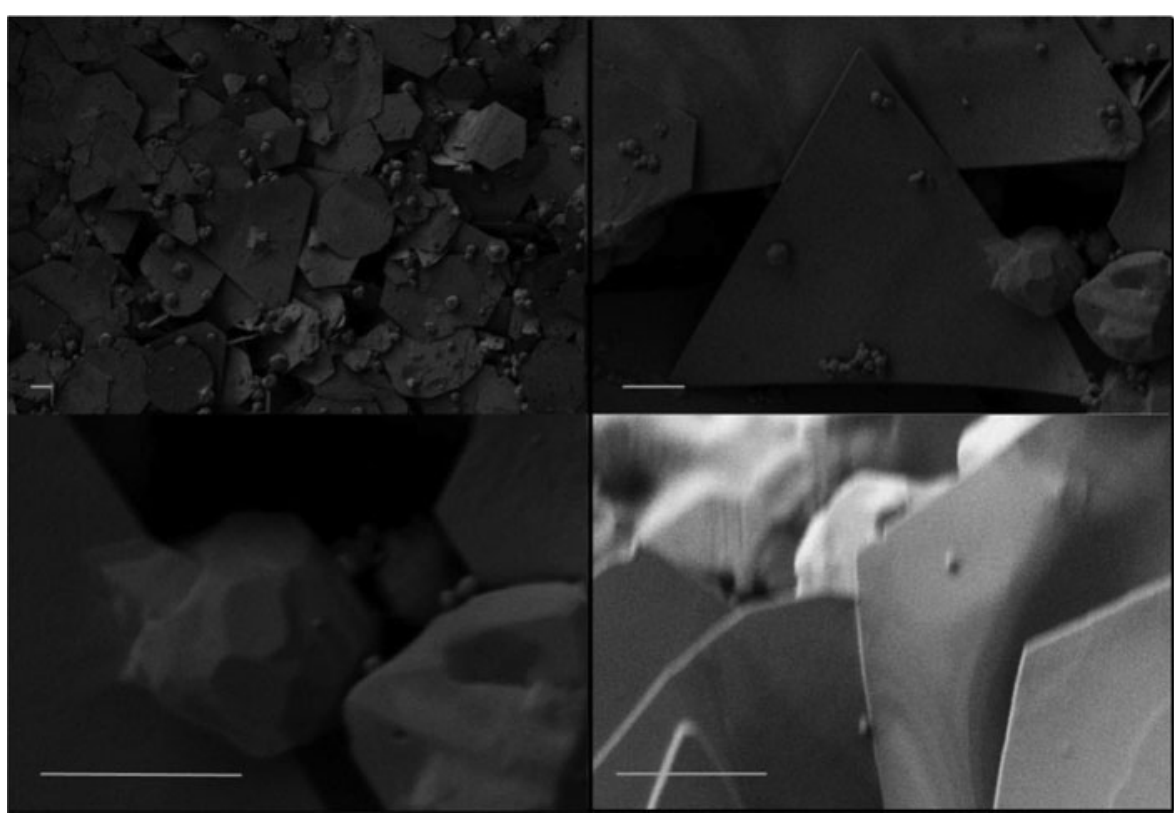

SUPPLEMENTARY FIG. S9. Representative SEM of GNP synthesized with gentamicin. Scale bar $=50 \mathrm{~nm}$. Replace "SF9" with "SF8" and rearrange its ubication 


\section{AUTHOR QUERY FOR END-2019-0686-VER9-ROCCA_1P}

AU1: Please identify (highlight or circle) all authors' surnames for accurate indexing citations.

AU2: Please mention all authors' degree abbreviations (e.g., MS, MD, PhD).

AU3: Please expand "C." in the author name "M. Jazmin Silvero C."

AU4: Please mention the location (city and state if in USA, or city and country if otherwise) in affiliations 2 and 3.

AU5: Please provide a short Tweet (120 characters including spaces) about your article that we can release upon publication (optional). Please email the Tweet to Paige Casey at Liebert's Marketing Department at PCasey@ liebertpub.com Please include your manuscript ID number (ex: end-2019-0686) in the subject heading of your email when responding to Ms. Casey.

AU6: Graphical abstract has been retained in article. Please confirm its inclusion.

AU7: Please provide the "conclusion text and credit line" for Graphical Abstract.

AU8: Please expand "C." in the author name "M. Jazmin Silvero C."

AU9: Please expand "RMN."

AU10: Please define "NMR."

AU11: Supplementary Figure S6 citation is not in sequential order in the text. Please check.

AU12: "M.J.S." has been changed as "M.J.S.C." per authors' group. Please check.

AU13: Ref. 9 is duplicates of Ref. 8. Hence duplicate entry has been deleted and references have been renumbered. Please check.

AU14: Please provide the date you last viewed the website for Ref. 2.

AU15: In Ref. 8, please mention the authors' names and article title.

AU16: Please provide the date you last viewed the website for Ref. 8.

AU17: Please mention the degree abbreviation (e.g., MS, MD, PhD) of the corresponding authors.

AU18: Please expand "C." in the author name "M. Jazmin Silvero C."

AU19: Please define "NMR." 